\title{
A Case of Successful Multi-Stage Open Surgical Conversion of a Secondary Aorto-Oesophageal Fistula with Endograft Infection After Thoracic Endovascular Aortic Repair
}

\author{
Youwen Chen*1, Junjie $\mathrm{Xu}^{2}$ and Zhijian Guo ${ }^{3}$ \\ ${ }^{1}$ Department of Cardiovascular Surgery, China \\ ${ }^{2}$ Department of Thoracic and Cardiovascular Surgery, Taiwan \\ ${ }^{3}$ Department of Thoracic Cardiovascular Surgery, China
}

Received: 制: December 31, 2018; Published: 制: January 11, 2019

*Corresponding author: Youwen Chen, Department of Cardiovascular Surgery, China

\begin{abstract}
Background Upper gastrointestinal bleeding (UGIB) is a major cause of morbidity and mortality accounting for approximately 56.5/100,000 hospitalizations with a mortality rate of $8.2 \%$. Secondary aorto-esophageal fistula (SAEF), a pathologic anatomical communication between the aorta and oesophagus in patients previously treated for thoracic aortic disease with a prosthetic graft, is a rare but life-threatening cause of massive UGIB. SAEF is uniformly fatal if untreated and remains a formidable surgical problem in older, high-risk patients with sepsis or hemorrhagic shock. An infected aortic aneurysm combined with SAEF is uncommon and considered to be one of the most challenging problems faced by cardiovascular surgeons. Thoracic endovascular aortic repair (TEVAR) is a minimally invasive alternative and is particularly valuable as a "bridging" procedure in emergency situations. However, TEVAR does nothing to address the issue of the defect in the digestive tract, which leaves these patients at high risk of aorto-oesophageal fistula (AEF) recurrence and/or endograft infection and leads to a high rate of re-intervention.
\end{abstract}

Open repair after TEVAR is performed in $2.2-7.2 \%$ of patients at experienced centres, and the associated morbidity and mortality can be significantly higher than with primary open repair. We described a successful multi-stage combined approach for a patient with SAEF and endograft infection. Case Presentation A 73-year-old Asian male was diagnosed with a proximal descending saccular aortic aneurysm with a surrounding haematoma at another hospital 97 days prior to presentation. The patient underwent TEVAR of the descending thoracic aorta (Zone 3) a week later and was discharged from hospital 4 days postoperatively. He was re-hospitalized for recurrent UGIB, persistent fever (average, $\sim 38.0-38.7^{\circ} \mathrm{C}$ ) with poststernal pain and difficulties in 54 days prior to being transferred. TEVAR-associated postoperative SAEF with para-aortic infection and delayed type Ia endoleak were confirmed, and the patient underwent 6 open surgical conversions. Perioperative antibiotic treatment was initiated with combined broad-spectrum antibiotics and later adjusted to tigecycline and colistin methanesulfonate. The patient was discharged on the $117^{\text {th }}$ postoperative day (1st surgery) with oral antibiotics. The patient's sternal wound had healed, and there were no clinical signs of infection based on radiological (chest X-ray) and laboratory examinations. However, the patient's long-term survival and prognosis still need to be monitored with continuous follow-up. Conclusions TEVAR for SAEF is associated with a high risk of recurrence of AEF, endograft infection and mediastinitis and should be utilized as a "bridge" to a definitive surgical repair.

Keywords: Gastrointestinal Bleeding; Aorto-Oesophageal Fistula; Thoracic Endovascular Aortic Repair; Endograft Infection; Mediastinitis; Endoleak

Abbreviations: UGIB: Upper Gastrointestinal Bleeding; SAEF: Secondary Aorto-Oesophageal Fistula; TEVAR: Thoracic Endovascular Aortic Repair; TAA: Thoracic Aortic Aneurysm; RTAD: Retrograde Type A Dissection; ER: Emergency Room; CT: Computed Tomography; BMI: Body Mass Index; AIDS: Acquired Immunodeficiency Syndrome; PE: Physical Examination; $\mathrm{FiO}_{2}$ : Fraction of Inspiration $\mathrm{O}_{2}$; PEEP: Positive End-Expiratory Pressure; SIMV: Synchronized Intermittent Mandatory Ventilation; VAC: Vacuum-Assisted Closure; CTA: Computed Tomography Angiography; EGD: Oesophagogastroduodenoscopy; DTA: Descending Thoracic Aorta; AsAo: Ascending Aorta; DsAo: Descending Aorta

\section{Introduction}

Upper gastrointestinal bleeding (UGIB) is a major cause of morbidity and mortalityaccounting for approximately 56.5/100,000 hospitalizations with a mortality rate of $8.2 \%$ [1]. Secondary aorto-oesophageal fistula (SAEF), a pathologic anatomical 
communication between the aorta and oesophagus in patients previously treated for thoracic aortic disease with a prosthetic graft, is a rare but life-threatening cause of massive UGIB that was first described by Chiari in 1914. The reported incidence of SAEF after surgery is low (4.8\%) [2]. SAEF is uniformly fatal if untreated and remains a formidable surgical problem in older, high-risk patients with sepsis or hemorrhagic shock. Thoracic aneurysm is the most common cause of AEF. As the thoracic aneurysm grows in size, the increasing tension on its wall erodes the aorta leading to rupture into the surrounding oesophagus and uncontrolled bleeding [3]. The classical clinical features of AEF are described as Chiari's triad, which comprises mid-thoracic pain, initial sentinel arterial hemorrhage and fatal exsanguination hours to days after an asymptomatic interval [4].

An infected aortic aneurysm combined with SAEF is rare and considered to be one of the most challenging problems faced by cardiovascular surgeons. Conservative treatment of SAEF is not a feasible option because most reported cases result in fatality [2]. The standard treatment of open surgery with resection of the infected aortic segment, extra-anatomic bypass or in situ reconstruction of the aorta, direct suturing for oesophageal repair or oesophageal resection with oesophagogastroplasty or coloplasty, and extensive debridement of the surrounding tissue carries significant morbidity and mortality rates (Figure 1). Operative and in-hospital mortality rates have been reported to be as high as $44 \%$ and $57 \%$, respectively, even at experienced centres [3-5]. Thoracic endovascular aortic repair (TEVAR), a less invasive option for patients with this serious condition, was first reported by Semba et al and has become one of the main therapies for thoracoabdominal aortic diseases including thoracic aortic aneurysm (TAA), traumatic aortic transection, and AEF. TEVAR is a minimally invasive alternative to the standard treatment and is especially valuable as a "bridging procedure" in emergency situations [5-6].

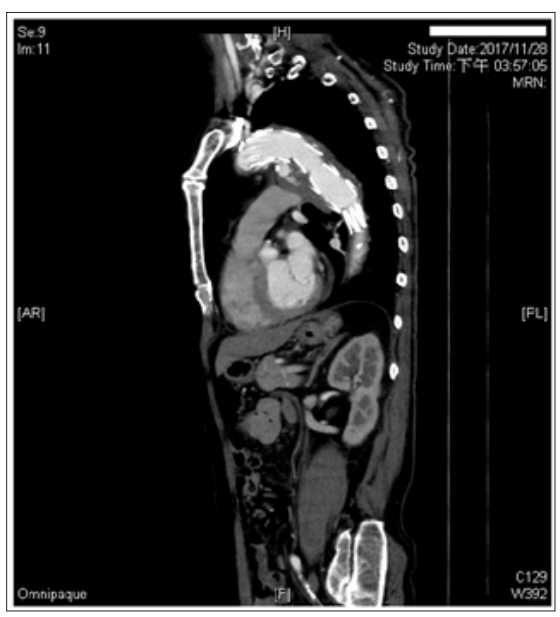

Figure 1: Enhanced chest CT examination was performed 3 days after admission to our hospital when the patient's general condition was stable and confirmed TEVARassociated postoperative SAEF with delayed type Ia endoleak and para-aortic infection.

TEVAR is associated with better early and late outcomes than open repair, including lower perioperative morbidity and mortality and has changed the therapeutic paradigm for thoracic aortic lesions. This difference in outcomes has led to TEVAR replacing open repair as the procedure of choice for most patients with TAAs and suitable anatomy [6]. TEVAR has recently gained popularity as an emergent treatment for AEF due to better perioperative outcomes and safety than open surgery in unstable patients. However, TEVAR does nothing to address the issue of the defect in the digestive tract, which leaves these patients at high risk of AEF recurrence and/ or stent graft infection and leads to a high rate of re-intervention. Re-intervention after TEVAR is reported in up to 3.8\% of patients independently of the type of endograft implanted (Figure 2). Although re-intervention is often endovascular, patients with serious complications (including device failure, endograft migration, endograft infection, endoleak, AEF or aorto-bronchial fistula and retrograde type A dissection (RTAD) that may lead to aneurysm rupture) often ultimately undergo conversion to open surgery $[3,7]$.

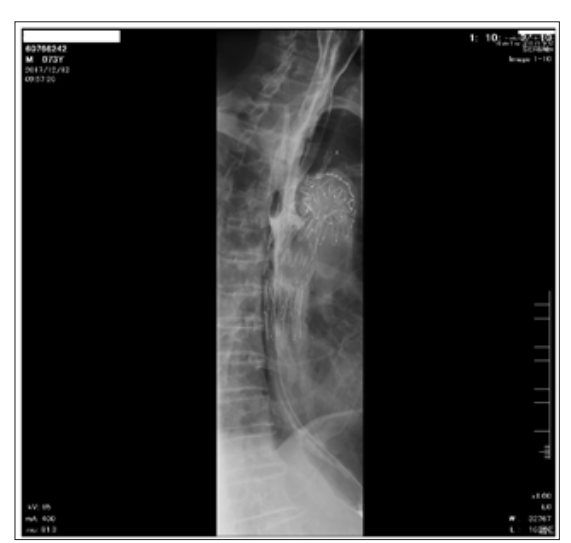

Figure 2: Oesophagography was performed before the second surgery. Apparent contrast agent leakage from the oesophagus into the DsAo during swallowing confirmed SAEF.

TEVAR has been performed more frequently despite the lack of randomized controlled trials proving the improved long-term effectiveness of TEVAR over open surgical repair. However, long-term endograft durability remains a concern $[3,6]$. As the number of TEVAR survivors has grown, reports of complications have similarly increased. The incidence of open repair after TEVAR ranges from $2.2 \%$ to $7.2 \%$ at experienced centres, and the associated morbidity and mortality can be significantly higher than with primary open repair [8]. The presence of the device leads to more technically challenging surgical exposure, and the patients are often considerably physiologically compromised. The development of strategies to manage patients with such complex conditions is absolutely imperative [5,7]. Herein, we described a case of the patient who emergently presented with SAEF and an endograft infection. We treated this patient with the use of a combined approach: TEVAR for the SAEF, followed by multi-stage surgeries to control infection of the aorta and correct the oesophageal lesion.

\section{Case Presentation}

A 73-year-old Asian male was diagnosed with a proximal descending saccular aortic aneurysm and a surrounding haematoma 
at another hospital 97 days prior to presentation. The patient underwent TEVAR of the descending thoracic aorta (Zone 3) a week later and was discharged from hospital 4 days after surgery. $\mathrm{He}$ was re-hospitalized for recurrent UGIB, persistent fever (average, $\sim 38.0-38.7^{\circ} \mathrm{C}$ ) with poststernal pain and difficulties in eating 54 days prior to being transferred to the emergency room (ER) of Kaohsiung Chang Gung Hospital. The patient was diagnosed with TEVAR-associated postoperative SAEF with massive haematemesis and delayed type Ia endoleak based on chest computerized tomography (CT) and clinical symptoms. He was treated with intravenous administration of cefuroxime $(1.5 \mathrm{~g} \mathrm{q} 8 \mathrm{~h}$ ) as antimicrobial therapy and had a jejunostomy tube placed to provide adequate nutritional replenishment. Twenty-two days before hospitalization in our hospital, a considerable para-aortic infection was noted on chest $\mathrm{CT}$, and the patient's intravenous antibiotic therapy was adjusted to cefuroxime ( $3.0 \mathrm{~g} \mathrm{q} 8 \mathrm{~h})$.

The patient progressed to having repeated massive haematemesis, tarry stool and fever, continuous poststernal pain, low oxygen saturation (<90\% with mask oxygen support), disturbances in arterial blood gas readings, difficulties in breathing requiring tracheal intubation and mechanical ventilation to prevent airway compromise and anaemia requiring homologous blood transfusions (Figure 3). The patient's clinical appearance and condition did not improve after aggressive conservative treatment, and he was transferred to our hospital for further surgical interventions. The patient was intubated and conscious when he was transferred to the ER of Kaohsiung Chang Gung Hospital with the abovementioned medical history. He was thin (167 centimetres in height, 47.8 kilograms in weight, body mass index (BMI) of $17.14 \mathrm{~kg} / \mathrm{m}^{2}$ ) and had anorexia and fatigue with fever $\left(38^{\circ} \mathrm{C}\right)$. The patient described himself ( and was described by his family members) as otherwise quite healthy, and he had never been admitted to a hospital before the TEVAR procedure 90 days prior. He reported a 10-year history of primary hypertension and a 7-year history of benign prostatic hyperplasia, and he had been taking oral amlodipine (5mgq12h), valsartan (160mgq12h), labetalol (200mg q12h) and silodosin (4mgq12h).

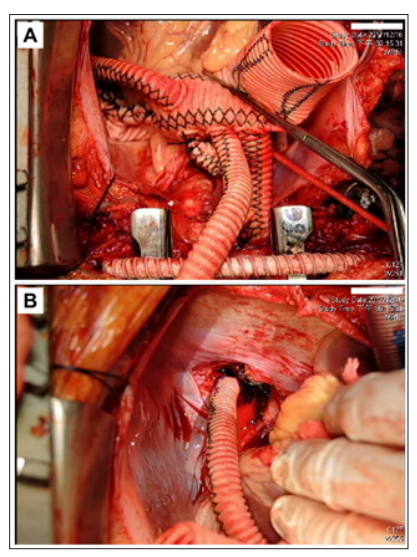

Figure 3: Interoperative image of the second surgery. Obliteration of the distal AsAo and innominate artery orifice and ligation of the distal DsAo were performed. Graft replacement of the AsAo and bypasses from the proximal four-branched aortic graft to the innominate artery and distal DTA were performed.
The patient reported no other significant chronic medical history, such as diabetes mellitus, any type of heart disease, disturbed microcirculation, peripheral neuropathy, immunocompromise, malignancies, leukaemia, long-term use of corticosteroids, liver cirrhosis, renal failure, urinary tract infection, or haemodialysis (Figure 4). He also reported no history of infection, such as tuberculosis, any type of hepatitis, or acquired immunodeficiency syndrome (AIDS). He had undergone TEVAR and several blood transfusions as previously mentioned, and he had no prior history of traumas or other serious events in his medical history. He did not live in an epidemic area and had no history of toxin or radiation exposure. He was previously a businessman and had been retired for 8 years. Physical examination (PE) revealed an elderly male who was conscious, awake and alert but in moderately painful distress with apparent generalized anxiety despite previous treatment with intravenous morphine (10mgq4hprn) and risperidone (1mghs). He was intubated with a fraction of inspiration $\mathrm{O}_{2}\left(\mathrm{FiO}_{2}\right)$ of $50 \%$ and a positive end-expiratory pressure (PEEP) of $7 \mathrm{cmH}_{2} \mathrm{O}$ under synchronized intermittent mandatory ventilation (SIMV) mode control.

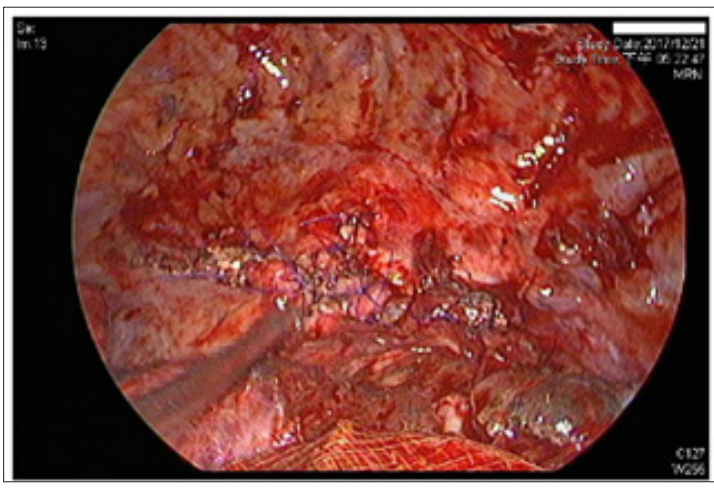

Figure 4: Interoperative image of the third surgery. Primary closure of oesophageal defect was performed with 4-0 Prolene in an interrupted suture pattern witSh a residual aortic granulation tissue buttress.

His respiratory rate was $18-26$ breaths/minute with an oxygen saturation of $97 \%$. His pulse was 86 beats/minute, his blood pressure was $102 / 73 \mathrm{mmHg}$, and his temperature was $38^{\circ} \mathrm{C}$. The examining physician noted mildly pale conjunctivae and slight bilateral basal crackles on chest auscultation. His breath pattern, breath sounds and thoracic percussion were documented as normal. No positive signs were found during integumentary, cardiovascular, abdominal and musculoskeletal examinations. An electrocardiogram revealed sinus rhythm with a normal axis. Laboratory findings revealed a normocytic anaemia. His red blood cell count, haemoglobin and haematocrit were $3.1 \times 10^{12} / \mathrm{L}, 10.2 \mathrm{~g} / \mathrm{dL}$ and $34.6 \%$, respectively. His white blood cell count was $17.1 \times 10^{9} / \mathrm{L}$, his ST-segment elevation was $81.0 \%$, and his C-reactive protein level was $75.3 \mathrm{mg} / \mathrm{L}$. Serology of both liver and renal functions was normal. TEVAR-associated postoperative SAEF with UGIB and delayed type Ia endoleak were considered based on a chest CT examination (provided by the previous hospital) and clinical symptoms.

The patient underwent a repeated enhanced chest CT examination with complete aortic three-dimensional reconstruction to 
elucidate the aorto-oesophageal situation after his general condition was stable 3 days after admission. SAEF with a considerable para-aortic infection and type Ia endoleak were confirmed, and the patient underwent 6 open surgical conversions as follows:

a) The first surgery was performed to achieve the primary goal of rapidly controlling bleeding and preventing fatal exsanguination on the 4th day of hospitalization. First, the right common carotid artery to the left common carotid artery and the right subclavian artery to the left subclavian artery were bypassed with a 6-mm seal polytetrafluoroethylene (PTFE) graft, TEVAR was performed with a Cook ZTEG-2PT-38-202-PF (over Zone 1) for SAEF, and embolization of the left subclavian orifice was performed with an 8-mm Amplatzer plug. Interoperative aortography did not showed any endoleak.

b) The second surgery was performed to excise the infected aorta with the previous endografts. Reconstruction of the aorta and complete debridement were performed on the $21^{\text {st }}$ postoperative day (after the first surgery) when the patient's clinical appearance was improved. Obliteration of the distal ascending aorta (AsAo) and innominate artery orifice and ligation of the distal descending aorta (DsAo) were performed. Integral debridement around the thoracic aorta and oesophagus was completed. Graft replacement of the AsAo and bypasses from the proximal four- -branched aortic graft (Gelseal, 28/10/8/8mm) to the innominate artery and distal descending thoracic aorta were also performed. The heart beat well after these procedures were performed, and the patient tolerated the whole surgery well.

c) The third surgery was performed with assistance from the department of thoracic surgery on the $26^{\text {th }}$ postoperative day after the patient was haemodynamically stable. The surgery was performed through a lateral thoracotomy with thoracoscopic guidance. The infected TAA was excised, and the previous thoracic aortic endografts were removed. A loculated haematoma (approximately 1200 millilitres of blood clots with turbid discharge in the left pleural cavity) was removed. Complete debridement of necrotic oesophageal tissue and mediastinum was performed followed by primary closure of the oesophageal defect (a $3 \times 4 \mathrm{~cm}$ erosion in the upper third of the oesophageal wall at the level of the aortic arch) with residual aortic granulation tissue and an intercostal muscle flap buttress by using 4-0 Prolene in an interrupted suture pattern. The DsAo above the supra-diaphragmatic level was then ligated. Finally, pneumonolysis of pleural adhesions and decortication of pleural peel were performed.

d) The fourth surgery was performed on the $45^{\text {th }}$ postoperative day due to active bleeding in the mediastinum. Rupture of the subclavian-subclavian bypass graft was considered because of extensive infection involving the upper mediastinum. The bleeding source was detected at a disrupted area of the upper chest subclavian-subclavian bypass graft. The graft was divided, and both ends were ligated doubly with 2-0 Prolene. No bleeding was noted after ligation. e) The fifth surgery was performed on the $51^{\text {st }}$ postoperative day because of mediastinitis. The bilateral subclavian PTFE grafts were removed, and the arteriotomy was closed with 5-0 Prolene followed by sequestrectomy of the sternum and integral debridement of the mediastinum. A sponge was placed over the mediastinum, and vacuum-assisted closure (VAC) was performed.

f) The sixth surgery was performed with assistance from the department of plastic surgery on the $6^{\text {th }}$ postoperative day for a chest wound infection with bone and soft tissue necrosis.

Sequestrectomy over the sternal bone and complete debridement of the wound were performed. Bilateral sides of the pectoris major were harvested for a myofasciocutaneous flap that was advanced to the defect (however, the middle part of the graft to cover the wound above the heart was too tight and was covered with muscle instead). Most of the chest wound was closed, and drainage with continuous lower-pressure suction was inserted $(18-25 \mathrm{mmHg})$. The patient was extubated successfully 3 days after the sixth surgery and transferred out of the cardiovascular surgery intensive care unit on the $89^{\text {th }}$ postoperative day. The sutured parts of the sternal wound was treated with topical neomycin and tyrothricin ointment, and the bandaging was changed twice per day until the skin was healed completely. The middle part of the sternal wound care was treated with Aquacell dressings that were changed once per day until healthy granulation tissue was observed. The patient's intravenous antibiotic therapy was cefuroxime (3gq8h) before admission.

Perioperative antibiotic treatment with combined broad-spectrum antibiotics (ceftazidime $1 \mathrm{~g} \mathrm{q} 12 \mathrm{~h}$ and tigecycline $0.05 \mathrm{~g} \mathrm{q} 12 \mathrm{~h}$ for 27 days) were initiated and later adjusted based on culture and sensitivity of the microbial isolates and empirical recommendations suggested by infectious disease specialists. The patient was therefore administered intravenous tigecycline $(0.05 \mathrm{~g} \mathrm{q} 12 \mathrm{~h})$ and colistin methanesulfonate $(0.167 \mathrm{~g} \mathrm{q} 12 \mathrm{~h})$ for 84 days. The patient's white blood cell count and C-reactive protein level gradually decreased. On the third postoperative day after the sixth surgery, his white blood cell, red blood cell and platelet counts were $10.7 \times 10^{9} / \mathrm{L}$, $3.24 \times 10^{12} / \mathrm{L}$ and $190 \times 10^{9} / \mathrm{L}$, respectively. His haemoglobin level was $107 \mathrm{~g} / \mathrm{L}$, his ST-segment elevation was $70.0 \%$, and his C-reactive protein level was $54.24 \mathrm{mg} / \mathrm{L}$. Serology of both liver and renal function was normal. He had no fever thereafter.

The patient was discharged on the $117^{\text {th }}$ postoperative day with antibiotics adjusted to oral administration of cefixime $(0.1 \mathrm{~g} \mathrm{q} 12 \mathrm{~h})$ for 4 weeks until there was no clinical bacteriological evidence or symptoms of ongoing infection. The patient was evaluated at the outpatient department twice for follow-up. The sternal wound healed, and there was no clinical evidence of infection based on the results of radiological (chest X-ray) and laboratory examinations. The patient was healthy as usual, with no complaints or illnesses. His complete cell counts and serum parameters of liver and kidney function were normal. He was continued on oral valsartan (160mg q12h), amlodipine (5mg q12h), and labetalol (200mg q12h) for hypertension and acetaminophen (500mg q8h) for pain relief. How- 
ever, the patient's long-term survival and prognosis still need to be monitored with continuous follow-up.

\section{Discussion}

AEF is an abnormal anatomical communication between the aorta and oesophagus. Dubrueil first described this condition in 1818 in a French soldier who ingested a beef rib and then had massive haematemesis [1]. Chiari described a triad of mid-thoracic pain, herald haemorrhage, and fatal haematemesis seen in AEF patients. AEFs are classified as primary if they are caused by spontaneous erosion of the aortic wall into the oesophagus and secondary if they occur as a complication following aortic or oesophageal surgery. Due to the increasing number of interventions involving the aorta, SAEF is 10 times more common than primary AEF $[1,4,8]$. Primary AEF and SAEF are rare but uniformly fatal without surgical intervention and remain a formidable surgical problem in older, high-risk patients with sepsis or haemorrhagic shock. The first report of successful surgical management of SAEF was described in 1978 by Smaha and colleagues [2,8]. In the past, infections such as tuberculosis and syphilis that lead to aortitis were the most common cause of AEF. In the current antibiotics era, the major causes for AEF formation include TAA, endovascular graft repairs, thoracic neoplasms, foreign body ingestion, advanced oesophageal cancer, radiofrequency ablation for atrial fibrillation, and surgical procedures involving the aorta and oesophagus $[3,4,9]$.

Other causes described in the literature are acid reflux disease, oesophageal biopsies and trauma. AEF after TEVAR is a rare unusual complication of TEVAR (incidence, 1.9\%), and the incidence of TEVAR failure requiring open surgical correction or additional open surgical procedures ranges from $2.2 \%$ to $7.2 \%$ [7,8]. Chiari's triad of chest pain, herald haemorrhage and sentinel haematemesis followed by massive haematemesis has been reported in the literature as a common characteristic presentation in patients with AEF. Other less frequent presentations include mediastinitis and/or fever. Complications of AEF include infections of the endovascular or surgical grafts, mediastinitis, sepsis, and death. Therefore, AEF can be a cause or a complication of endograft infection $[1,8]$. Our patient presented with the typical Chiari's triad and a continuous fever, and we considered that infection of the endovascular and surgical grafts and mediastinitis were complications caused by SAEF. Computed tomography angiography (CTA) and oesophagogastroduodenoscopy (EGD) are two common methods used to diagnose AEF.

However, the sensitivity of EGD for identifying the source of bleeding in AEF associated UGIB is $<10 \%$. EGD may show a pulsatile mass, bluish discoloration, or simply blood clotting in the oesophagus in one-third of patients. CTA is diagnostic in most cases and shows active extravasation of contrast material from the aorta to the oesophagus $[6,8]$. However, AEF should be suspected based on the following findings on CT following TEVAR: mediastinal air, air in the aortic lumen, persistent or expanding perigraft fluid, soft tissue density beyond 6 months from the date of the procedure, or decreased aorto-oesophageal fat plane $[6,8]$. As an initial diagnostic, an enhanced chest CT examination with complete aortic three-dimensional reconstruction can also provide enough information to elucidate the aorto-oesophageal situation. Any strategy in patients with AEF needs to be highly individualized given the risks of major thoracic aortic surgery that often performed in a complex operative field. A multicentre survey conducted in Italy showed that results were better with a combined approach in which TEVAR was followed by definitive open surgical repair than with TEVAR alone (overall mortality of $25 \%$ in combined cases versus $52.5 \%$ after TEVAR alone).

The recurrence rate of SAEF after TEVAR alone was reported to be $20 \%$ versus $6.2 \%$ in after combined TEVAR with open repair during a mean follow-up for 7.4 months $[1,4]$. Therefore, AEF should be treated with emergent aortic stenting to control bleeding once diagnosed, and TEVAR is mostly recommended as a "bridging procedure" for stabilizing the patients in extremis, followed by effective debridement (in addition broad-spectrum antibiotics and antifungals, repeated thorough mediastinal debridement is required for adequate infection control owing to contamination from the gastrointestinal tract, as one-quarter of endografts become infected) and more extensive reconstruction of the descending thoracic aorta (DTA) and oesophagus at a later stage [2]. Complex aortic and oesophageal reconstruction is performed in multiples stages once the infection is controlled. The perioperative outcomes of the endovascular approach have demonstrated favourable outcomes. Growing experience in TEVAR currently helps surgeons to identify anatomical or clinical situations associated with a high risk of endograft failure $[2,3,5]$.

TEVAR has recently emerged as an alternative treatment to open surgery for SAEF, especially in unstable patients or those who are poor surgical candidates. TEVAR offers a less invasive option and allows prompt exclusion of the SAEF thus preventing bleeding and rapidly facilitating haemodynamic stabilization. However, concerns regarding the durability of TEVAR even in the short-term are broadly realistic. Studies have reported significant in-hospital mortality after TEVAR alone, secondary to high rates of re-infection, mediastinitis, sepsis, and SAEF recurrence [2]. TEVAR alone leaves the oesophageal defect untreated and the endograft is directly exposed to a contaminated environment and ongoing infection. Growing evidence suggests that TEVAR for AEF does nothing to address the issue of the defect in the oesophageal, which leaves patients at risk of AEF recurrence and/or endograft infection, persistent mediastinitis and sepsis $[3,8]$. Despite significant refinement of surgical techniques and even when a diagnosis of an SAEF is reached, the morbidity and mortality are high, reaching up to $43 \%$, especially in patients with comorbidities or a general health status that initially precluded open surgical repair $[3,6]$.

Several options for AEF surgical repair have been well described in the literature. The conventional open surgical approach, involving combined replacement of the proximal DTA (in situ reconstruction using antibiotic-soaked grafts or a homograft) and/ or bypass of the thoracic aorta with concomitant resection of the infected aortic endograft and oesophageal repair (via direct suturing or oesophageal diversion with postponed reconstruction) or resection (with oesophagogastroplasty or coloplasty) with coverage 
using muscle or pleural flaps, is associated with high intraoperative and perioperative mortality rates, ranging from $45.4 \%$ to $55 \%$. Other have described an extra-anatomic prosthetic bypass from the AsAo to the distal DsAo or the supraceliac aorta with excision of the infected graft $[3,8]$. In contrast with in situ reconstruction, this extra-anatomic approach has a decreased potential for graft re-infection and reduces the total ischemic time in patients who are already hemodynamically compromised [2]. In our patient, we performed an extra-anatomic bypass from the AsAo to the distal DsAo to minimize the risks of re-infection, followed by resection of the previous infected aortic endografts and definitive repair of the oesophageal perforation via a primary repair.

The oesophageal repair was reinforced with a pedicled muscle flap as a buffer to minimize recurrent infections and enteric connections. Combined immediate or staged surgical procedures were significantly associated with decreased aortic-related mortality in a univariate analysis $[3,6,8]$. The advantage of the combined approach is to address the two essential aims of the surgical procedure: first, TEVAR allows minimally invasive and rapid control of the bleeding; second, the open repair allows surgical debridement of the infected mediastinum with reconstruction of the aortic wall and repair of the oesophageal defect $[3,7]$. Surgical repair of an SAEF with considerable endograft infection is a complex procedure that often requires extracorporeal circulation and adjunctive modalities to provide organ protection to prevent neurological, myocardial, and visceral complications [7]. With the increasing use of TEVAR, more patients will present with emergency indications for surgical correction; thus, knowledge regarding TEVAR conversion is mandatory. The time between the two stages has to be determined according to markers of malnutrition, such as subjective global assessment, history of weight loss, and transport proteins (albumin, alpha-fetoprotein, vitamin D-binding protein).

Patients should be referred for open surgery as soon as possible once they are clinically improved $[3,4]$. We recognized that TEVAR is a good emergency alternative to prevent early fatal exsanguination in patients with AEF. However, TEVAR can rarely be used as a stand-alone procedure because the oesophageal defects is left untreated, increasing the risks of sepsis, mediastinitis and death. In the present case, we performed a repeated TEVAR procedure $\left(1^{\text {st }}\right.$ operation), bypass of the right-left common carotid artery and subclavian artery with $6 \mathrm{~mm}$ SEAL PTFE grafts, and embolization of the left subclavian orifice with an $8 \mathrm{~mm}$ Amplatzer plug. Accordingly, we decided to treat the oesophageal lesion when the patient was in stable condition. We believed that the best treatment was direct suturing of the oesophageal defects with residual aortic granulation tissue and an intercostal muscle flap. This was feasible because the defect was small enough for direct repair (using 4-0 Prolene in an interrupted suture pattern), and the surrounding oesophageal walls were neither grossly contaminated nor extensively necrotic.

The use of intercostal muscle flaps to protect and strengthen the sutured oesophageal lesions arose from the understanding that striated muscle is better suited than pericardial or omental fat for closing contaminated wounds. A recent study involving 47 patients with AEF showed that open surgical repair of the aorta (with a prosthesis or homograft) and oesophagus with increased intercostal muscle coverage significantly improved the mid-term mortality rates [10]. Antibiotic treatment is the only strongly negative factor associated with a lower aortic mortality rate in a multivariate analysis. The underlying aetiology of AEF predicts long-term survival, and infectious and malignant aetiologies are associated with the worst outcomes $[9,10]$. Therefore, antibiotic treatment should be applied after endovascular stent placement to correct and prevent potential thoracic endograft infection. Postoperative antibiotic strategies differed widely among the various manuscripts, both with regard to the choice of antibiotic and the duration of therapy. Empirical broad-spectrum antibiotics, including antifungal coverage, are appropriate initially $[9,10]$.

One could argue for 6- to 8-week courses of broad-spectrum intravenous antibiotics followed by life-long oral antibiotic use for those treated with TEVAR alone. Some physicians insist that lifelong treatment with oral suppressive antibiotics is required, while others claim that such treatment can be discontinued provided there is no clinical bacteriological or radiological evidence of ongoing sepsis [3]. Our patient was diagnosed with TEVAR-associated postoperative AEF with massive haematemesis and delayed type Ia endoleak at another hospital 54 days prior to being transferred to our hospital and was treated with intravenous cefuroxime $1.5 \mathrm{~g}$ q8h). Twenty-two days before hospitalization at Kaohsiung Chang Gung Hospital, considerable para-aortic infection was identified on chest CT, and the patient's intravenous antibiotic therapy was adjusted to cefuroxime ( $3 \mathrm{~g} \mathrm{q} 8 \mathrm{~h}$ ). However, after a few weeks of reinforced medication, the patient continued to have a fever and several episodes of massive haematemesis and tarry stool. After 6 open surgical repairs performed in our hospital, perioperative antibiotic treatment with combined broad-spectrum antibiotics (ceftazidime $1 \mathrm{~g} \mathrm{q} 12 \mathrm{~h}$ and tigecycline $0.05 \mathrm{~g} \mathrm{q} 12 \mathrm{~h}$ for 27 days) was initiated and later adjusted based on culture and sensitivity of the microbial isolates and empirical recommendations suggested by infectious disease specialists (tigecycline $0.05 \mathrm{~g} \mathrm{q} 12 \mathrm{~h}$ and colistin methanesulfonate $0.167 \mathrm{~g} \mathrm{q} 12 \mathrm{~h}$ for 84 days).

The patient was discharged with oral antibiotics (cefixime $0.1 \mathrm{~g} \mathrm{q} 12 \mathrm{~h}$ for 4 weeks) until there was no clinical bacteriological evidence or symptoms of ongoing infection. We have found that patient compliance with life-long antibiotics is critical to achieve a good outcome. Cessation of oral antibiotics may cause re-infection or (surgical) graft infection, although the necessity of life-long antibiotics in cases of this nature is still debated. Our current plan for patients with this condition is continuous antibiotics after there are no clinical indications of infection based on the results of radiological and laboratory examinations. Importantly, TEVAR does not definitively repair the AEF and should be used as a "bridge procedure" until a definitive surgical intervention is performed. Recent studies have shown an increased risk of mediastinitis and recurrent fatal bleeding and a high mortality rate if TEVAR is used as the sole treatment modality. Hence, all patients who have undergone TEVAR should be closely followed up, and definitive surgical correction of the aorta and the oesophagus should be considered when they are deemed to be in appropriate health for surgery $[6,7]$. A nationwide, 
multicentre survey performed in Italy yielded information on 25 aorto-oesophageal and aorto-bronchial fistulae that were treated with TEVAR.

The data revealed that patients who undergo combined treatment (TEVAR+ surgical oesophageal/ bronchial repair) may have lower graft infection rates and better survival rates than patients who simply receive TEVAR alone [2]. We are firmly convinced that the long-term prognosis is strictly affected by graft contamination and mediastinal infection. To minimize these events in patients, we administer broad-spectrum antibiotics therapy; the specific medications choices are guided by available culture results, and treatment decisions are made in collaboration with infectious disease specialists. Due to the low incidence of this disease entity, it is unlikely that a planned prospective randomized study will be conducted. However, a large prospective registry may help to establish the precise indications and benefits of TEVAR and the eventual need for a multi-stage approach $[2,10]$. A multidisciplinary approach is advisable to achieve prompt control of bleeding, using TEVAR first, sometimes an extra-anatomic bypass from the AsAo to the distal the DsAo, total excision of the infected aortic endograft and/or surgical graft, reconstruction of the aortic continuity, repair of the oesophageal defect, and appropriate antibiotic therapy. These are the founation of managing patients with SAEF. The present case demonstrates that a multi-stage approach involving a multidisciplinary team can improve patient survival.

\section{Conclusion}

From the presenting case, we learned that in certain clinical or anatomic situations, caution should be recommended when performing endovascular procedures in patients with thoracic aortic diseases. TEVAR for SAEF is associated with a high risk of recurrence of AEF, endograft infection and mediastinitis and should be utilized as a "bridge" to a definitive surgical repair. Open conversion of a failed thoracic endograft is a complicated and difficult procedure. Surgical repair can be offered and have encouraging results if performed by a multidisciplinary team experienced in the management of thoracic aortic diseases. With the increasing use of TEVAR, more patients will present with indications for surgical correction; thus, knowledge regarding TEVAR conversion is mandatory given that the procedure is being performed in patients with more challenging anatomy and broader indications $[1,9]$.

\section{References}

1. Rana OA, Harith HM, Harleen KS, Khalil K, Safi HJ, et al. (2016) Successful multistaged surgical management of secondary aortoesophageal fistula with graft infection. Ann Thorac Surg 101(6): 203-205.

2. Ludovic C, Pierre A, Thomas G, Albat B, Marty Ané C, et al. (2011) Surgical conversion after thoracic endovascular aortic repair. J Thorac Cardiovasc Surg 142(5): 1027-1031.

3. Canaud L, Alric P, Gandet T, Ozdemir BA, Albat B, et al. (2013) Open surgical secondary procedures after thoracic endovascular aortic repair. Eur J of Vasc and Endovasc Surg 46(6): 667-674.

4. Ludovic C, Baris AO, William WB, Bahia S, Holt P, et al. (2014) Thoracic endovascular aortic repair in management of aortoesophageal fistulas. J Vasc Surg 59(1): 248-254.

5. Boonprasit K, Dhanakom P, Jitpreedee S, Tantarattanapong W, Rookkapan S, et al. (2011) Endovascular therapy for infected aortic aneurysms. J Vasc Surg 54(5): 1259-1265.

6. Majid AA, Fahad B, Abdulrahman MA (2013) Fatal aortoesophageal fistula bleeding after stenting for a leak post sleeve gastrectomy. World J Gastrointest Surg 5(12): 337-340.

7. Laxmi K, Mohan R (2015) Primary aortoesophageal fistula: great masquerade of esophageal variceal bleeding. Indian J of Critical Care Medicine 19(2): 119-121.

8. Seifeldin H, Mihajlo G, Lohit G, Molly Orosey, Tusar Desai (2016) Atypical aortoesophageal fistula with atypical and delayed presentation and negative imaging studies. Case Reports in Gastrointestinal Medicine.

9. Malav PP, Muhammed S, Sreelakshmi P, Harsh A Rawal, Venu Gopalakrishnan (2016) Radiation therapy-induced aortoesophageal fistula: a case report and review of literature. Gastroenterology Report $4(2): 165-167$.

10. Enrico MM, Giovanni C, Andrea K, Yamume Tshomba, Roberto Chiesa (2010) Combined endovascular and surgical treatment of primary aortoesophageal fistula. Tex Heart Inst J 37(6): 722-724.
ISSN: 2574-1241

DOI: 10.26717/BJSTR.2019.13.002349

Youwen Chen. Biomed J Sci \& Tech Res

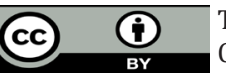

This work is licensed under Creative Commons Attribution 4.0 License

Submission Link: https://biomedres.us/submit-manuscript.php

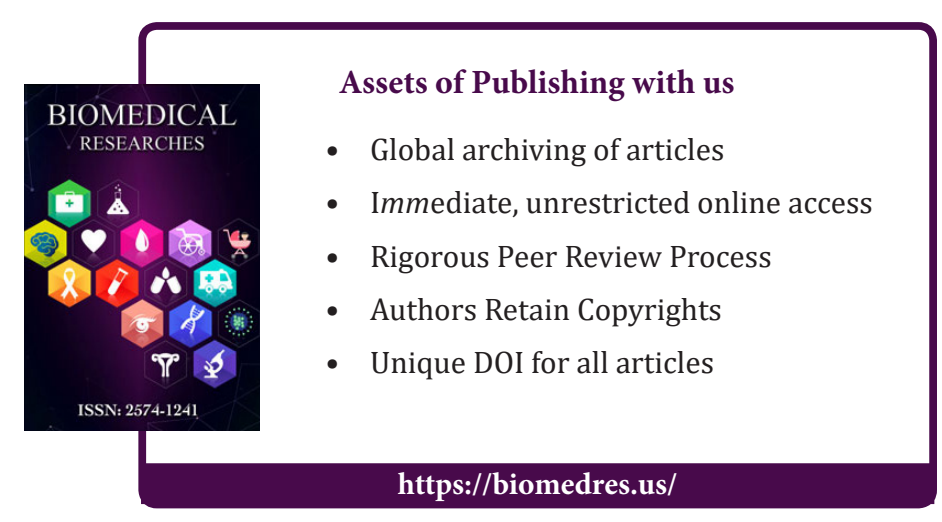

